\title{
Effect of lift force on the aerodynamics of dust grains in the protoplanetary disk
}

\author{
Masaki S Yamaguchi ${ }^{*}$ and Shigeo S Kimura ${ }^{2}$
}

\begin{abstract}
We newly introduce lift force into the aerodynamics of dust grains in a protoplanetary disk. Although many authors have investigated the effects of the drag force, gravitational force, and electric force on the dust grains, the lift force has never been considered as a force exerted on dust grains in a gas disk. We show in this paper that the dust grains can be continuously spinning as a result of the frequent collisions and that the lift force continues to be exerted on them, which is valid in a certain parameter space where the grain size is larger than approximately $1 \mathrm{~m}$ and where the distance from the central star is larger than $1 \mathrm{AU}$ for the minimum mass solar nebula. In addition, we estimate the effects of the force on the grain motion and obtain results that show that the mean relative velocity between the grains due to the lift force is comparable to the gas velocity in the Kepler rotational frame when the Stokes number and lift-drag ratio are both approximately 1. This estimation is performed under the assumptions of steady state and the isotropic spin angular momentum. We also estimate the mean relative velocity when the grains keep spinning and conclude that the lift force marginally affects the mean relative velocity in the minimum mass solar nebula. If there is a grain-concentrated part in the disk, the relative velocity due to the lift force may dominate there because of the high collision rate.
\end{abstract}

Keywords: Gas disk; Protoplanetary; Aerodynamics; Dust grains

\section{Background}

In the theory of planet formation, the issue concerning the radial drift of meter-size dust remains an open question. In a typical scenario, micrometer-size dust grains grow to be kilometer-size planetesimals via collision and merging in a protoplanetary disk (Goldreich and Ward 1973; Hayashi 1981). When the dust grain grows to be metersized, there is velocity with respect to the disk gas and losses in the angular momentum due to the drag force. Thus, the grain falls down to the central star and cannot grow further (Adachi et al. 1976; Brauer et al. 2008).

Various scenarios have been proposed for solving the issue of meter-size dust. The gravitational instability in the dust layer was investigated first (Goldreich and Ward 1973; Sekiya 1983). In this scenario, the dust grains settle toward the mid-plane to form the dense layer, which then fragments into precursors of the planetesimals. However, the sedimentation of the grains leads to vertical shear

*Correspondence: masaki.yamaguchi@nao.ac.jp

1 National Astronomical Observatory, 2-21-1 Osawa, Mitaka, Tokyo 181-8588, Japan

Full list of author information is available at the end of the article of the rotational velocity in the dust layer, which causes turbulence due to the Kelvin-Helmholtz instability. As a result, the grains cannot settle enough to form planetesimals (Sekiya 1998). The effects of turbulence due to magneto-rotational instability have also been considered (Balbus and Hawley 1976; Sano et al. 2000). Collisions between dust grains occur more frequently when there is an increase of the relative velocity due to the turbulence, so the growth rate of the grains also increases. Brauer et al. (2008), Okuzumi et al. (2012), and Kataoka et al. (2013) have taken into account these effects of the turbulence, and while the first study has found that the dust grains fall down to the central star if the grain density is relatively large, the second and third ones have found that the dust grains can grow at a sufficiently rapid rate to avoid the issue of meter-size dust when the grains are fluffy. As another scenario Youdin and Goodman (2005) have suggested, the planetesimals may be formed by the streaming instability caused by the interaction between the dust grains and disk gas.

We newly introduce lift force as a factor affecting the relative velocity between the dust grains. When a grain

\section{是 Springer}

(c) 2014 Yamaguchi and Kimura; licensee Springer. This is an Open Access article distributed under the terms of the Creative Commons Attribution License (http://creativecommons.org/licenses/by/4.0), which permits unrestricted use, distribution, and reproduction in any medium, provided the original work is properly credited. 
moves in fluid and when the fluid around the grain has circulation, the lift force is exerted on the grain perpendicularly to the grain velocity, and this is generally represented as

$$
F_{\mathrm{L}}=C_{\mathrm{L}} \cdot \frac{\pi r_{\mathrm{d}}^{2}}{m_{\mathrm{d}}} \cdot \frac{1}{2} \rho_{\mathrm{g}}|\vec{u}|^{2},
$$

where $C_{\mathrm{L}}, r_{\mathrm{d}}, m_{\mathrm{d}}, \rho_{\mathrm{g}}$, and $\vec{u}$ represent the coefficient of the lift force, the radius and the mass of the grain, the density of the gas, and the velocity of a dust grain relative to the disk gas, respectively. We note here that $F_{\mathrm{L}}$ is defined as the lift force per unit mass. The coefficient of the lift force is determined by properties of the grain and flow. For a rotating sphere, the lift force is expressed as

$$
\vec{F}_{\mathrm{L}}=\frac{\pi \rho_{\mathrm{g}} r_{\mathrm{d}}^{3}}{m_{\mathrm{d}}} \vec{\omega}_{\mathrm{d}} \times \vec{u},
$$

where $\vec{\omega}_{\mathrm{d}}$ represents the vector of the angular velocity of the grain. In Equation (2), Stokes law, which is valid when the Reynolds number is small, is adopted as the drag law (Rubinow and Keller 1961; Takagi 1974). In this case, the coefficient of the lift force is represented as

$$
C_{\mathrm{L}}=\frac{2 m_{\mathrm{d}} F_{\mathrm{L}}}{\pi r_{\mathrm{d}}^{2} \rho_{\mathrm{g}} u^{2}}=\frac{2 r_{\mathrm{d}} \omega_{\mathrm{d}}}{u} \sin \theta,
$$

where $\theta$ is the angle between $\vec{\omega}_{\mathrm{d}}$ and $\vec{u}$. When the Reynolds number is so large that the turbulent flow is dominant, and when the Knudsen number is so large that the fluid cannot be regarded as a continuum, the lift force has not yet been formulated. Therefore, in this paper, we investigate the effects of the lift force only when Equation (2) can be applied. We estimate the conditions under which the lift force continues to be exerted on the grain in the 'Sustainability of the spin of the dust grains' section. We derive and reduce an equation of motion for the grain to estimate the relative velocity between the grains in the 'Relative velocity between the dust grains' section. In the 'Discussion' section, we evaluate the relative velocity between the grains when the lift force is kept exerted on the grains and discuss potential areas of improvement for our model. Finally, we summarize our study in the 'Conclusions' section.

\section{Methods and results}

\section{Sustainability of the spin of the dust grains}

In this section, we examine whether the dust grains keep spinning in the gas disk because the lift force does not act on non-spinning spherical objects. Here, we assume that the collisions between the dust grains induce the spin of the dust grains. The spinning dust is subjected to the torque due to the friction created by the background viscous fluid. After the spin-down timescale, the spin of the dust would stop. We can estimate the collision time $t_{\text {col }}$ and the spin-down time $t_{\text {spin-down }}$. By comparing these timescales, we obtain the parameter space where the lift force can act on the spinning dust grains. These timescales depend on the disk structure. We adopt the parameters for the disk structure in this paper as follows:

$$
\begin{aligned}
\Sigma_{\mathrm{g}} & =\Sigma_{0} R_{1}^{-q}, \\
\Sigma_{\mathrm{d}} & =f_{\mathrm{d}} \Sigma_{\mathrm{g}}=f_{\mathrm{d}} \Sigma_{0} R_{1}^{-q}, \\
c_{\mathrm{s}} & =\sqrt{\frac{k T}{\bar{m}}}=c_{\mathrm{s}, 0} R_{1}^{-p}, \\
\Omega_{\mathrm{K}} & =\sqrt{\frac{G M_{\mathrm{s}}}{R^{3}}}=\Omega_{0} R_{1}^{-3 / 2}, \\
\nu_{\mathrm{K}} & =R \Omega_{\mathrm{K}}=\Omega_{0} R_{0} R_{1}^{-1 / 2}, \\
H_{\mathrm{g}} & =\frac{\sqrt{2} c_{\mathrm{s}}}{\Omega_{\mathrm{K}}}=\frac{\sqrt{2} c_{\mathrm{s}, 0}}{\Omega_{0}} R_{1}^{-p+3 / 2}, \\
\rho_{\mathrm{g}} & =\frac{\Sigma_{\mathrm{g}}}{\sqrt{\pi} H_{\mathrm{g}}}=\frac{\Sigma_{0} \Omega_{0}}{\sqrt{2 \pi} c_{\mathrm{s}, 0}} R_{1}^{p-q-3 / 2},
\end{aligned}
$$

where $R$ is the semi-major axis, $R_{0}$ is the typical radius of the disk, $R_{1}=R / R_{0}, f_{\mathrm{d}}$ is the dust-to-gas mass ratio, $\bar{m}=$ $2.35 m_{H}$ is the mean particle mass of gas, and $M_{\mathrm{S}}=1 M_{\odot}$ is the mass of the central star. We use the isothermal sound speed $c_{\mathrm{S}}$ and the mid-plane gas density $\rho_{\mathrm{g}}$ when estimating timescales. If we choose $\Sigma_{0}=1.7 \times 10^{3} \mathrm{~g} \mathrm{~cm}^{-2}, c_{\mathrm{s}, 0}=$ $1.0 \times 10^{5} \mathrm{~cm} \mathrm{~s}^{-1}, \Omega_{0}=2.0 \times 10^{-7} \mathrm{~s}^{-1}, R_{0}=1 \mathrm{AU}, f_{\mathrm{d}}=$ $0.01, q=3 / 2$, and $p=1 / 4$, the disk profile is similar to the minimum mass solar nebula (MMSN; Hayashi 1981).

\section{Collision timescale}

The collision timescale is estimated as

$$
t_{\mathrm{col}} \sim\left(n_{\mathrm{d}} \cdot \pi r_{\mathrm{d}}^{2} \cdot<v_{\mathrm{d}-\mathrm{d}}>\right)^{-1},
$$

where $n_{\mathrm{d}}$ and $v_{\mathrm{d}-\mathrm{d}}$ are the number density of the dust grains and the relative velocity between the dust grains, respectively. The parenthetic quantity $<Q>$ represents the statistical average.

The dust number density is expressed as

$$
n_{\mathrm{d}}=\frac{\Sigma_{\mathrm{d}}}{H_{\mathrm{d}} m_{\mathrm{d}}}
$$

where $H_{\mathrm{d}}$ is the scale height of the dust layer and $m_{\mathrm{d}}$ is the mass of the dust grains. We approximate that the mass distribution function of the dust is the delta function because it is necessary for the dust grains to collide with similar scale grains so that the grains gain the angular momentum. Considering the equilibrium between turbulent diffusion and sedimentation (Birnstiel et al. 2010), $H_{\mathrm{d}}$ is obtained as

$$
H_{\mathrm{d}}=H_{\mathrm{g}} \cdot\left(\frac{\alpha}{\mathrm{St}} \frac{1+2 \mathrm{St}}{1+\mathrm{St}^{2}}\right)^{1 / 2},
$$

where St $\equiv \Omega_{\mathrm{K}} t_{\mathrm{S}}$ is the Stokes number ( $t_{\mathrm{s}}$ is the stopping time by drag force). We use alpha prescription $v_{\text {turb }}=$ $\alpha c_{\mathrm{s}} H_{\mathrm{g}}$ to describe the strength of the turbulence in the 
protoplanetary disk and assume $\alpha<$ St to avoid the situation $H_{\mathrm{g}}<H_{\mathrm{d}}$. At the Stokes drag law regime, the Stokes number is written as

$$
\mathrm{St}=\frac{m_{\mathrm{d}} \Omega_{\mathrm{K}}}{6 \pi r_{\mathrm{d}} \rho_{\mathrm{g}} v}=\frac{2 \rho_{\mathrm{int}} r_{\mathrm{d}}^{2} \Omega_{\mathrm{K}}}{3 \rho_{\mathrm{g}} c_{\mathrm{s}} \lambda_{\mathrm{mfp}}}=\frac{2 \sigma_{\mathrm{mol}} \rho_{\mathrm{int}} \Omega_{0}}{3 \bar{m} c_{\mathrm{s}, 0}} r_{\mathrm{d}}^{2} R_{1}^{p-3 / 2},
$$

where $\rho_{\text {int }}$ is the internal mass density of the dust grains, $v=c_{\mathrm{s}} \lambda_{\mathrm{mfp}} / 3$ is the kinematic viscosity, and $\lambda_{\mathrm{mfp}}$ is the mean free path of the gas particles. The mean free path is estimated as $\lambda_{\mathrm{mfp}}=\bar{m} /\left(\sigma_{\mathrm{mol}} \rho_{\mathrm{g}}\right)$, where $\sigma_{\mathrm{mol}}$ is the cross section of collisions between $\mathrm{H}_{2}$ molecules. We adopt $\rho_{\text {int }} \simeq 3 \mathrm{~g} \mathrm{~cm}^{-3}$ and $\sigma_{\mathrm{mol}} \simeq 2 \times 10^{-15} \mathrm{~cm}^{2}$. Equation (14) means that the Stokes number is independent of the normalization coefficient of the surface density $\Sigma_{0}$, which is canceled out because of the one in $\lambda_{\mathrm{mfp}}$.

Since the gas was assumed to be in a turbulent state described by the alpha prescription, we set the mean relative velocity $<v_{\mathrm{d}-\mathrm{d}}>=<v_{\mathrm{d}-\mathrm{d}}>_{t}$, where $<v_{\mathrm{d}-\mathrm{d}}>_{t}$ means the relative velocity between the grains in the turbulent gas. According to (Ormel and Cuzzi 2007), $\left.<v_{\mathrm{d}-\mathrm{d}}\right\rangle_{t}$ with similar scale grains can be represented as

$$
<v_{\mathrm{d}-\mathrm{d}}>_{t}=c_{\mathrm{S}}\left(\frac{\alpha \mathrm{St}}{\sqrt{1+\frac{1}{4} \mathrm{St}^{2}(1+S t)^{2}}}\right)^{1 / 2},
$$

where we smoothly interpolate the two limiting solutions of St $\gg 1$ and St $\ll 1$. This expression is valid when the stopping time is larger than the turnover time of the Kolmogorov-scale eddy. The minimum size of the grain satisfying this condition is on the order of sub-millimeters for MMSN at $1 \mathrm{AU}$; hence, we focus on grains larger than approximately $1 \mathrm{~mm}$ in what follows.

Now we can express $t_{\mathrm{col}}$ as the function of $r_{\mathrm{d}}$ and $R_{1}$ by using Equations (11) to (15) as

$$
t_{\mathrm{col}}=\frac{4 \sqrt{2} \rho_{\mathrm{int}}}{3 \Omega_{0} \Sigma_{0} f_{\mathrm{d}}} r_{\mathrm{d}} R_{1}^{q+3 / 2} f(\mathrm{St}),
$$

where

$$
f(\mathrm{St})=\frac{1}{\mathrm{St}}\left(\frac{1+2 \mathrm{St}}{1+\mathrm{St}^{2}}\right)^{1 / 2}\left(1+\frac{1}{4} \mathrm{St}^{2}(1+\mathrm{St})^{2}\right)^{1 / 4} .
$$

We note that when $\alpha<\mathrm{St}, t_{\mathrm{col}}$ is independent of $\alpha$ because the effect of increasing $<v_{\mathrm{d}-\mathrm{d}}>_{t}$ balances the one decreasing $n_{\mathrm{d}}$. For the case with $\mathrm{St} \ll 1$, the collision timescale is $t_{\text {col }} \propto \mathrm{St}^{-1} r_{\mathrm{d}}^{1} R_{1}^{q+3 / 2} \propto r_{\mathrm{d}}^{-1} R_{1}^{3+q-p}$, while it is $t_{\text {col }} \propto \mathrm{St}^{-1 / 2} r_{\mathrm{d}}^{1} R_{1}^{q+3 / 2} \propto r_{\mathrm{d}}^{0} R_{1}^{2.25+q-0.5 p}$ for the case with St $\gg 1$. If we adopt the same parameters as MMSN, the collision time is

$$
t_{\mathrm{col}}=1.7 \times 10^{6} f(\mathrm{St}) R_{1}^{3} r_{\mathrm{d}, 1} \mathrm{~s},
$$

where $r_{\mathrm{d}, 1}=r_{\mathrm{d}} /(1 \mathrm{~cm})$.

\section{Spin-down timescale}

In the case of Stokes law, the angular momentum conservation around the spin axis of a spherical grain is given as

$$
I_{\mathrm{d}} \frac{d \omega_{\mathrm{d}}}{d t}=-8 \pi \rho_{\mathrm{g}} v r_{\mathrm{d}}^{3} \omega_{\mathrm{d}}
$$

where $I_{\mathrm{d}}$ is the moment of inertia of the grain. The torque acting onto a spherical body by viscous fluid is given in Rubinow and Keller (1961) and Takagi (1974). From this equation, the $t_{\text {spin-down }}$ is estimated as

$$
t_{\text {spin-down }}=\frac{I_{\mathrm{d}}}{8 \pi \rho_{\mathrm{g}} v r_{\mathrm{d}}^{3}}=\frac{\rho_{\mathrm{int}} r_{\mathrm{d}}^{2}}{5 \rho_{\mathrm{g}} c_{\mathrm{s}} \lambda_{\mathrm{mfp}}}=\frac{\sigma_{\mathrm{mol}} \rho_{\mathrm{int}}}{5 \bar{m} c_{\mathrm{s}, 0}} r_{\mathrm{d}}^{2} R_{1}^{p} .
$$

In this second equation, we assume a spherical and uniform density grain whose moment of inertia is represented as $I_{\mathrm{d}}=2 m_{\mathrm{d}} r_{\mathrm{d}}^{2} / 5$. The spin-down time becomes longer as the dust grain becomes larger. Here, we note that the spin-down time is independent of $\Sigma_{0}$ for the same reason as the Stokes number [see Equation (14)]. For MMSN, $t_{\text {spin-down }}$ is estimated as

$$
t_{\text {spin-down }}=3.0 \times 10^{3} r_{\mathrm{d}, 1}^{2} R_{1}^{1 / 4} \mathrm{~s} .
$$

\section{Comparison of timescales}

Now, we can obtain the size of dust grains that are able to keep spinning. We estimate these timescales just in the Stokes law regime because the lift force in other regimes is uncertain. There are two necessary conditions to realize Stokes law. One is that the gas can be regarded as a continuum medium, which is expressed as $r_{\mathrm{d}} \gtrsim 9 \lambda_{\mathrm{mfp}} / 4$. The other is that the flow around the dust grains is laminar, which is represented as $\operatorname{Re}=2 u r_{\mathrm{d}} / v \lesssim 20$ (Shirayama 1992), where $u$ is the relative velocity of the dust to the gas. Here, we should actually include the effect of turbulence in the expression of $u$ as in Ormel and Cuzzi (2007) so that the physical situation is consistent with that of Equation (15). However, taking this effect into account causes complicated equations. Thus, as a firststep attempt, we assume that $u$ is equal to the relative velocity between the orbital velocity of the gas and the Keplerian velocity, i.e., $u=\eta v_{\mathrm{K}}$, where

$$
\eta \equiv \frac{2 p+2 q+3}{4}\left(\frac{c_{\mathrm{s}}}{v_{\mathrm{K}}}\right)^{2}
$$

which is given in (Adachi et al. 1976). By these conditions, we find that our estimation is valid in the following range:

$$
\begin{aligned}
r_{\mathrm{d}, \min } & \equiv \frac{9 \bar{m} c_{\mathrm{s}, 0}}{2 \sigma_{\mathrm{mol}} \Sigma_{0} \Omega_{0}} R_{1}^{q-p+3 / 2} \lesssim r_{\mathrm{d}} \lesssim r_{\mathrm{d}, \max } \\
& \equiv \frac{40 \sqrt{2 \pi} \bar{m} R_{0}}{3(2 p+2 q+3) \sigma_{\mathrm{mol}} \Sigma_{0}} R_{1}^{q+1} .
\end{aligned}
$$


For MMSN, this condition is simply written as

$$
3.2 R_{1}^{11 / 4} \lesssim r_{\mathrm{d}, 1} \lesssim 89 R_{1}^{5 / 2} \text {. }
$$

Figure 1 shows the two timescales $t_{\mathrm{col}}$ (solid lines) and $t_{\text {spin-down }}$ (dashed lines) at $R_{1}=1$ for MMSN. We plotted $t_{\text {col }}$ and $t_{\text {spin-down }}$ in the range that satisfies the condition (24). From Figure 1, we can see that $t_{\mathrm{col}}$ is larger than $t_{\text {spin-down }}$, so that the spin of the dust would stop at $R_{1}=1$. The difference between the two timescales gets smaller as the dust grains become larger. Equations (18) and (21) show that large grains are likely to satisfy the condition $t_{\text {col }}<t_{\text {spin-down. }}$. From Equation (24), the Stokes regime can be adopted for the larger dust grains at the outer region of the disk. Thus, we expect that the condition $t_{\text {col }}<t_{\text {spin-down }}$ is satisfied at the outer region $R_{1}>1$. Figure 2 shows the parameter space where the dust grains keep spinning in the $R_{1}-r_{\mathrm{d}}$ plane for MMSN. The Stokes regime is realized between the dashed green lines. The condition $t_{\text {col }}<t_{\text {spin-down }}$ is satisfied above the solid red line. In the blue region, the condition is satisfied with the Stokes regime. There are dust grains that keep spinning with the Stokes regime in $R_{1} \gtrsim 1.3$. The grains that can keep spinning have the size $r_{\mathrm{d}} \sim r_{\mathrm{d} \text {,max }}$. The dotted magenta line shows the dust radius when $\mathrm{St}=1$, which is used in the 'Discussion' section.

\section{Relative velocity between the dust grains}

In this section, we investigate whether the mean relative velocity is comparable to or greater than the gas velocity in the Kepler rotational frame. Since this gas velocity is comparable to the typical relative velocity between a large grain and a small one compared to 1-m-sized dust, we take it as a reference value. First, we derive the equation of motion for a dust grain assuming that it moves at a terminal velocity. Next, we estimate the mean relative velocity by assuming an isotropic distribution for the spin angular momentum.

Here, for simplicity, we assume that the dust grains move on the mid-plane of the disk, which means that the $z$-component of the lift force is assumed to be zero, where the $z$-axis is taken as the disk axis, and we adopt the cylindrical coordinates described below. Since the direction of the spin angular momentum can be taken arbitrarily, the lift force can show the $z$-component. Nevertheless, we neglect the $z$-component of the velocity to simplify the calculation below.

For preparation to derive the equation of motion, we express a projected vector of the lift force on the midplane in terms of the direction of the spin angular momentum of a dust grain. Since the direction of the lift force is perpendicular to the spin angular momentum and the velocity of the grain with respect to the gas, then

$$
\vec{F}_{\mathrm{L}}=A \vec{\omega}_{\mathrm{d}} \times \vec{u},
$$

where the coefficient satisfies $A=\frac{\pi \rho_{\mathrm{g}} \mathrm{r}_{\mathrm{d}}^{3}}{m_{\mathrm{d}}}$ (see the 'Background' section). Since the $z$-component of $\vec{u}$ is zero,

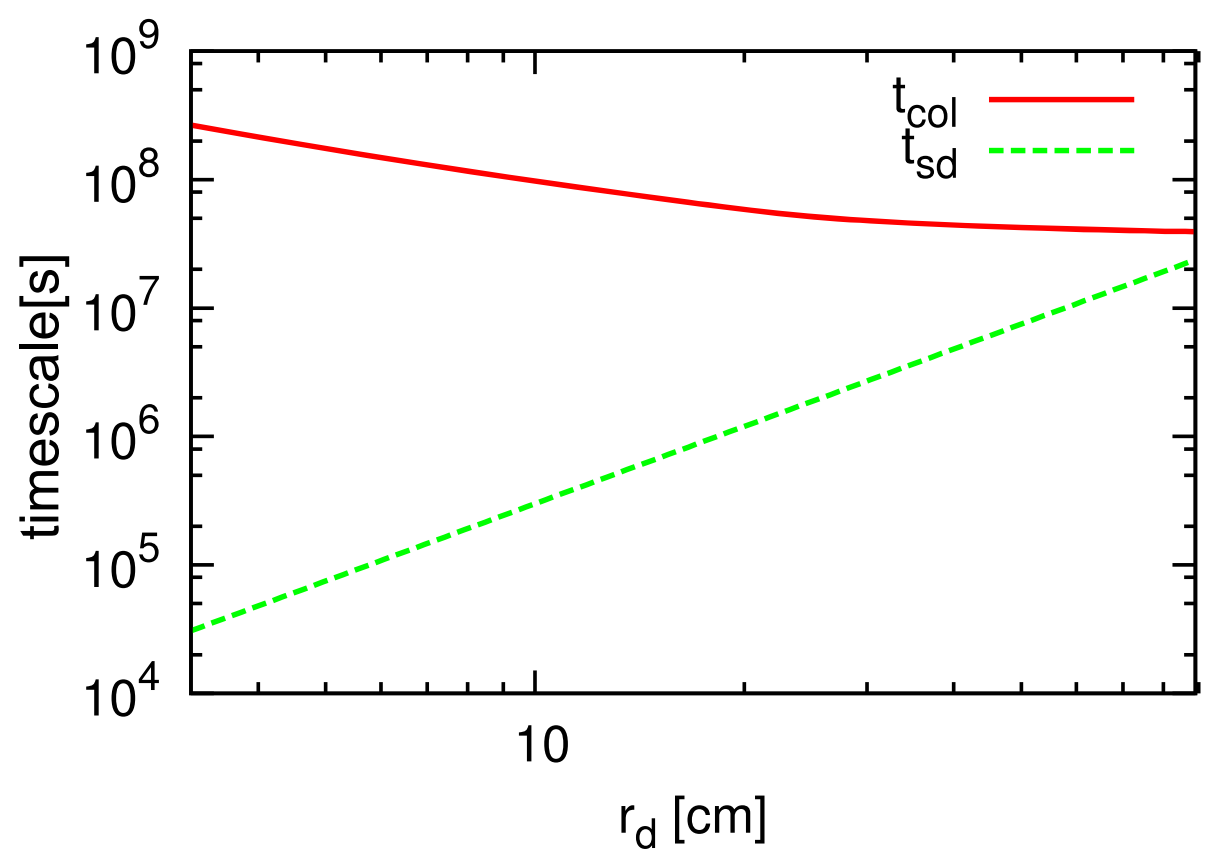

Figure $1 r_{\mathrm{d}}$ dependence of $\boldsymbol{t}_{\mathrm{col}}$ (solid lines) and $\boldsymbol{t}_{\text {spin-down }}$ (dashed lines) at $\boldsymbol{R}_{\mathbf{1}}=\mathbf{1}$. $t_{\mathrm{col}}$ is always longer than $t_{\text {spin-down }}$ in the Stokes regime. 


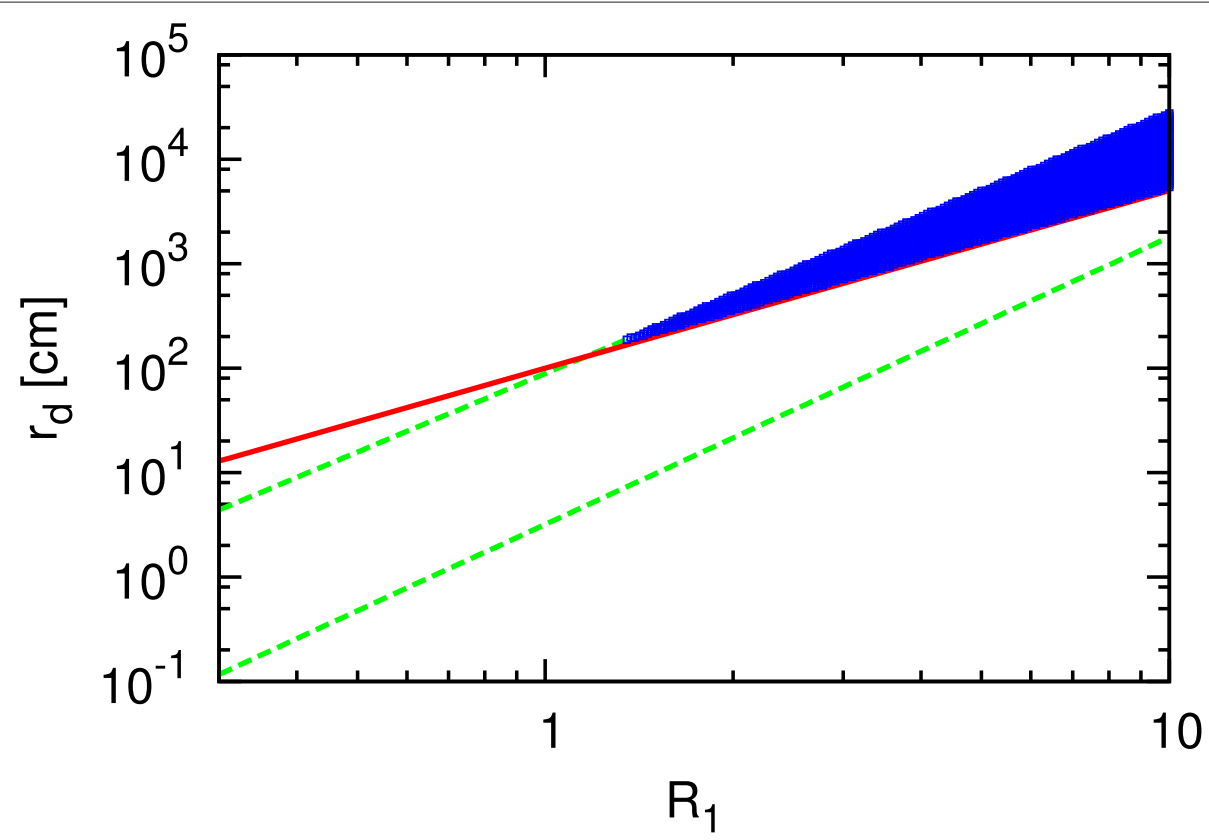

Figure 2 Parameter space where the dust grains can keep spinning in the $\boldsymbol{R}_{\mathbf{1}}-\boldsymbol{r}_{\mathrm{d}}$ plane. The Stokes regime is between the two dashed green lines. The condition $t_{\mathrm{col}}<t_{\text {spin-down }}$ is satisfied above the solid red line. The grains may keep spinning in the blue region. The dotted magenta line represents the line where the condition $S t=1$ is satisfied.

the lift force vector projected on the mid-plane $\vec{F}_{\mathrm{L} \text {,mid }}$ is expressed as

$$
\vec{F}_{\mathrm{L}, \text { mid }}=\vec{F}_{\mathrm{L}} \cdot\left(\begin{array}{c}
\vec{e}_{r} \\
\vec{e}_{\theta}
\end{array}\right)=A \omega_{\mathrm{d}} \mu\left(\begin{array}{c}
-u_{\theta} \\
u_{r}
\end{array}\right) \equiv F_{\mathrm{L}}\left(\begin{array}{c}
-u_{\theta} / u \\
u_{r} / u
\end{array}\right),
$$

where $\vec{e}$ with a subscript and $\mu$ represent a unit vector in the direction of the subscript and the cosine of the angle between $\vec{\omega}_{\mathrm{d}}$ and the $z$-axis, respectively. We note here that the $F_{\mathrm{L}}$ values do not depend on the azimuth angle of the spin angular momentum.

Next, we derive and reduce the equation of motion for a dust grain. Now, the forces exerted on the dust grain are the gravitational force of the central star, the drag force, and the lift force, so the equation of motion is expressed as

$$
\frac{d \vec{v}}{d t}=-\frac{G M_{\odot}}{r^{2}} \vec{e}_{r}-\vec{F}_{\mathrm{D}}+\vec{F}_{\mathrm{L}, \mathrm{mid}}
$$

where we assume that the mass of the central star is the same as the solar one. As the first step for the reduction of Equation (27), we divide it into two equations for the $r$ and $\theta$ components. Since the velocity of the disk gas does not have the radial component, the components of the velocity of the dust grain are represented as $\left(u_{r}, u_{\theta}\right)=$ $\left(v_{r}, v_{\theta}-r \Omega_{\mathrm{g}}\right)$, where $\Omega_{\mathrm{g}}$ is the orbital angular velocity of the disk gas around the central star. Thus, Equation (27) is expressed as

$$
\begin{aligned}
& \frac{d v_{r}}{d t}-\frac{v_{\theta}^{2}}{r}=-\frac{G M_{\odot}}{r^{2}}-F_{\mathrm{D}} \frac{v_{r}}{u}-F_{\mathrm{L}} \frac{v_{\theta}-r \Omega_{\mathrm{g}}}{u}, \\
& \frac{d v_{\theta}}{d t}+\frac{v_{r} v_{\theta}}{r}=-F_{\mathrm{D}} \frac{v_{\theta}-r \Omega_{\mathrm{g}}}{u}+F_{\mathrm{L}} \frac{v_{r}}{u} .
\end{aligned}
$$

As the second step, we transform this into coordinates rotating at the angular velocity of the Kepler rotation, that is, $v_{\theta}=v_{\mathrm{K}}+v_{\theta}^{\prime}$. As the third step, we assume that the motion of the dust grain is stationary and that $\left|v_{r}\right|,\left|v_{\theta}^{\prime}\right| \ll$ $\nu_{\mathrm{K}}$.

This stationary assumption may be invalid when taking into account the timescales discussed in the 'Sustainability of the spin of the dust grains' section. The stopping time $t_{\mathrm{s}}$ is represented as

$$
t_{\mathrm{s}}=\frac{m_{\mathrm{d}}}{6 \pi r_{\mathrm{d}} \rho_{\mathrm{g}} \nu} \sim 10^{4} r_{\mathrm{d}, 1}^{2} R_{1}^{1 / 4} \sim 4 t_{\mathrm{spin}-\text { down }} .
$$

This means that the dust grain stops spinning before moving at the terminal velocity independently of the dust size and the distance from the central star. Thus, as long as the lift force is exerted on the grain, the motion of the grain cannot reach a steady state. Alternatively, the grain motion can be considered to be determined by the merger of the parent grains (or scattering by the other grains). Nevertheless, we assume that the grain motion reaches the steady state for the first stage of this type of work. 
Finally, we non-dimensionalize the variables as

$$
x=\frac{v_{r}}{\eta v_{\mathrm{K}}}, y=\frac{v_{\theta}^{\prime}}{\eta v_{\mathrm{K}}}, g_{\mathrm{D}}=\frac{F_{\mathrm{D}}}{u \Omega_{\mathrm{K}}}, g_{\mathrm{L}}=\frac{F_{\mathrm{L}}}{u \Omega_{\mathrm{K}}},
$$

where $\eta$ is the constant satisfying the equation $r \Omega_{\mathrm{g}}=$ $\nu_{\mathrm{K}}(1-\eta)$ and $\Omega_{\mathrm{K}}$ is the angular velocity of the Kepler motion. Thus, we obtain two algebraic equations:

$$
\begin{gathered}
2 y=g_{\mathrm{D}} x+g_{\mathrm{L}}(y+1), \\
\frac{1}{2} x=-g_{\mathrm{D}}(y+1)+g_{\mathrm{L}} x .
\end{gathered}
$$

These equations represent the balance between the Coriolis, drag, and lift forces.

The solution of the equations is

$$
\begin{gathered}
x=\frac{-2 g_{\mathrm{D}}}{g_{\mathrm{D}}^{2}+\left(g_{\mathrm{L}}-2\right)\left(g_{\mathrm{L}}-1 / 2\right)}, \\
y=-1+\frac{-2\left(g_{\mathrm{L}}-1 / 2\right)}{g_{\mathrm{D}}^{2}+\left(g_{\mathrm{L}}-2\right)\left(g_{\mathrm{L}}-1 / 2\right)} .
\end{gathered}
$$

Here, Equations (26) and (31) lead to $g_{\mathrm{L}}=g_{\mathrm{L}, \max } \mu$, where $g_{\mathrm{L}, \max } \equiv \frac{A \omega_{\mathrm{d}}}{\Omega_{\mathrm{g}}}$, and we introduce the lift-drag ratio $R_{\mathrm{LD}} \equiv$ $g_{\mathrm{L}, \max } / g_{\mathrm{D}}$ to obtain $g_{\mathrm{L}}=g_{\mathrm{D}} R_{\mathrm{LD}} \mu$. Thus, $x$ and $y$ are expressed as functions of $\mu, g_{\mathrm{D}}$, and $R_{\mathrm{LD}}$. We show $x(\mu)$ and $y(\mu)$ in Figure 3 . Here, we assume $g_{\mathrm{D}}=1$ and $R_{\mathrm{LD}}=1$ as trial values.
Since $x=-1$ when we neglect the lift force, the curve of $x(\mu)$ shows that the radial velocity of the dust grain can be a third or four times of that without the lift force. On the other hand, when $\mu<0, y$ is almost constant and comparable to that without the lift force. When $\mu$ is larger than $0.5, y$ is smaller than -1 , which means that the dust grain orbits more slowly than the gas. In addition, we see that $x$ and $y$ decrease as $\mu$ is close to unity. Therefore, the absolute value of the velocity tends to increase as $\mu$ increases.

Next, we calculate the average and dispersion of the velocity of the dust grain on the disk mid-plane, assuming that the spin angular momentum is isotropic, which is just for simplicity. Thus, the direction distribution satisfies $f(\Omega)=\frac{1}{4 \pi}$, which is equivalent to $f(\mu)=\frac{1}{2}$, where $\Omega$ is a solid angle parameter. The average and dispersion of $x$ are calculated by performing the integration below.

$$
\begin{aligned}
<x> & =\int x f(x) d x \\
& =\frac{1}{2} \int_{-1}^{1} x(\mu) d \mu \\
<x^{2}> & =\int x^{2} f(x) d x \\
& =\frac{1}{2} \int_{-1}^{1} x^{2}(\mu) d \mu,
\end{aligned}
$$

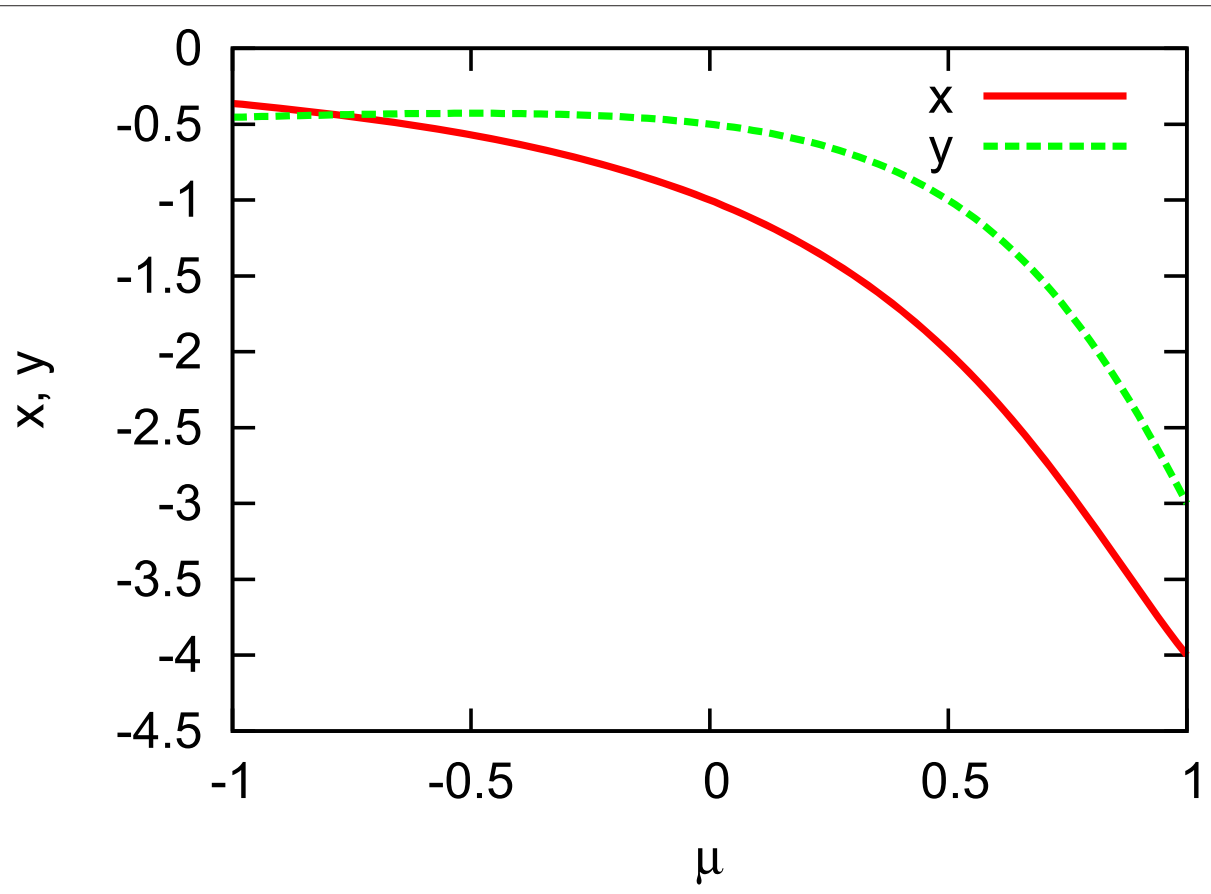

Figure 3 Dependence of the grain velocity on the direction of the spin angular momentum. We show the grain velocity in the radial direction $x$ (the solid line) and in the azimuthal direction $y$ (the dashed line). The variable $\mu$ represents the cosine of the angle between the spin angular momentum and $z$-axis. We take the parameters as $g_{\mathrm{D}}=1$ and $R_{\mathrm{LD}}=1$. 
where we transform the integration variable into $\mu$. We also can derive the same expression for $y$,

$$
\begin{aligned}
<y> & =\frac{1}{2} \int_{-1}^{1} y(\mu) d \mu, \\
<y^{2}> & =\frac{1}{2} \int_{-1}^{1} y^{2}(\mu) d \mu .
\end{aligned}
$$

By taking $g_{\mathrm{D}}=1$ and $R_{\mathrm{LD}}=1$, we can obtain the approximate value of the average and standard deviation of the velocity,

$$
\begin{aligned}
&<x> \simeq-1.4, \\
&<y>\simeq-1.2, \\
& \sqrt{<x^{2}>} \simeq 1.0, \\
& \sqrt{<y^{2}>} \simeq 0.6, \\
&<w_{\text {rel }}>\equiv \sqrt{<x^{2}>+<y^{2}>} \simeq 1.2 .
\end{aligned}
$$

Equation (38) means that the dust grains, on average, fall down to the star faster than without the lift force. Moreover, Equation (39) means that their average orbit is at almost the same velocity as the gas. We note that the standard deviation of the velocity represents the average of the relative velocity between the grains. Therefore, Equation (42) means that the average relative velocity exceeds the relative velocity between the gas and Kepler velocity, so that the collision rate is affected by the lift force when $g_{\mathrm{D}}=1$ and $R_{\mathrm{LD}}=1$.

We finally calculate the averaged relative velocity on the disk mid-plane $\left\langle w_{\text {rel }}>\right.$ for arbitrary values of $g_{\mathrm{D}}$ and $R_{\mathrm{LD}}$. Figure 4 shows the contour lines of $\left\langle w_{\text {rel }}\right\rangle=1$ (the solid line) and 0.1 (the dashed line) on the $g_{\mathrm{D}}-R_{\mathrm{LD}}$ plane. We see that the relative velocity is large when $g_{\mathrm{D}}$ is small and when $R_{\mathrm{LD}}$ is large, which corresponds to the situation where the lift force is efficiently exerted on the grains. The important fact is that there exists a region satisfying $<w_{\text {rel }}>>1$, where the lift force non-negligibly affects the dynamics of the system of grains, compared to the case without the force.

\section{Discussion}

\section{Lift-drag ratio}

In this subsection, we estimate the lift-drag ratio $R_{\mathrm{LD}}$ to investigate how efficiently the lift force affects the motion of the dust. In the Stokes regime, the lift coefficient $C_{\mathrm{L}}$ and drag coefficient $C_{\mathrm{D}}$ are represented as

$$
\begin{aligned}
C_{\mathrm{D}} & =\frac{4 c_{\mathrm{s}} \lambda_{\mathrm{mfp}}}{r_{\mathrm{d}} u}, \\
C_{\mathrm{L}} & =\frac{2 m_{\mathrm{d}} F_{\mathrm{L}}}{\pi r_{\mathrm{d}}^{2} \rho_{\mathrm{g}} u^{2}}=\frac{2 r_{\mathrm{d}} \omega_{\mathrm{d}}}{u} \sin \theta .
\end{aligned}
$$

The lift coefficient depends on the spin angular velocity $\omega_{\mathrm{d}}$ of the dust. Here, we estimate $\omega_{\mathrm{d}}$ induced by the collisions of the grains. When two grains with the same mass $m_{\mathrm{d}}$ collide with the impact parameter $b$, the angular momentum around the center of mass is represented by

$$
L=\frac{b v_{\mathrm{d}-\mathrm{d}} m_{\mathrm{d}}}{2} \text {. }
$$

Given the weight by a cross section, we derive the averaged angular velocity $\sqrt{<L^{2}>}$ as

$$
\sqrt{<L^{2}>}=\left(\frac{\int_{0}^{2 r_{\mathrm{d}}} L^{2} 2 \pi b d b}{\int_{0}^{2 r_{\mathrm{d}}} 2 \pi b d b}\right)^{1 / 2}=\frac{1}{\sqrt{2}}<v_{\mathrm{d}-\mathrm{d}}>r_{\mathrm{d}} m_{\mathrm{d}} .
$$

If we assume that the grain obtains the mass $2 m_{\mathrm{d}}$ and this angular momentum after the collision, the resultant angular velocity $\omega_{\mathrm{d}}$ is represented as

$$
\omega_{\mathrm{d}}=\frac{5 \sqrt{2}}{4} \frac{<v_{\mathrm{d}-\mathrm{d}}>}{r_{\mathrm{d}}} \text {. }
$$

Using Equations (43), (44), and (47), we can reduce the lift-drag ratio $R_{\mathrm{LD}}$ as

$$
R_{\mathrm{LD}}=\frac{C_{\mathrm{L}}}{C_{\mathrm{D}}}=\frac{5 \sqrt{2} r_{\mathrm{d}}<v_{\mathrm{d}-\mathrm{d}}>}{8 c_{\mathrm{s}} \lambda_{\mathrm{mfp}}} .
$$

Furthermore, we adopt $<v_{\mathrm{d}-\mathrm{d}}>=<v_{\mathrm{d}-\mathrm{d}}>_{t}$, so that this expression is nearly independent of the dust radius $r_{\mathrm{d}}$ for St $>1$. For St $\gg 1$, the lift-drag ratio approaches asymptotically to a maximum,

$$
R_{\mathrm{LD}} \simeq 20 \alpha^{1 / 2} R_{1}^{-17 / 8},
$$

where we take the MMSN disk parameters.

We see from Figure 2 that when $r_{\mathrm{d}}$ is approximately $10^{2} \mathrm{~cm}$ at $R_{1} \sim 1$, which corresponds to $g_{\mathrm{D}}=\mathrm{St}^{-1} \sim 0.1$, then the conditions where $t_{\mathrm{col}} \sim t_{\text {spin-down }}$ and the drag force is represented with Stokes law are marginally satisfied. In this case, we find $R_{\mathrm{LD}}$ of approximately 2.0 when $\alpha=0.01$ using Equation (49). Thus, from Figure 4, we obtain $<w_{\text {rel }}>\sim 0.1$, which means that the averaged relative velocity due to the lift force is a tenth of $\eta v_{\mathrm{K}}$ for the 1 -m-sized dust at $1 \mathrm{AU}$ from the central star.

\section{Dependence of the relative velocity on disk parameters}

The situation stated in the previous subsection can be qualitatively or quantitatively changed by adopting disk parameters different from MMSN. If we take an $f_{\mathrm{d}}$ larger than 0.01, as proposed in Sekiya (1998) and Hasegawa and Tsuribe (2014), the averaged relative velocity $<w_{\text {rel }}>$ can be approximately 1 . The dust-to-gas ratio $f_{\mathrm{d}}$ is included just in the expression of $t_{\mathrm{col}}$ [Equation (16)], so that a larger $f_{\mathrm{d}}$ means a smaller $t_{\mathrm{col}}$ and thereby smaller $r_{\mathrm{d}}$ satisfying $t_{\mathrm{col}}=t_{\text {spin-down }}$. This implies that the red line in Figure 2 moves down and that the blue region expands inside. When $f_{\mathrm{d}}$ is approximately 0.1 , the blue region includes the dotted magenta line, which shows the grain 


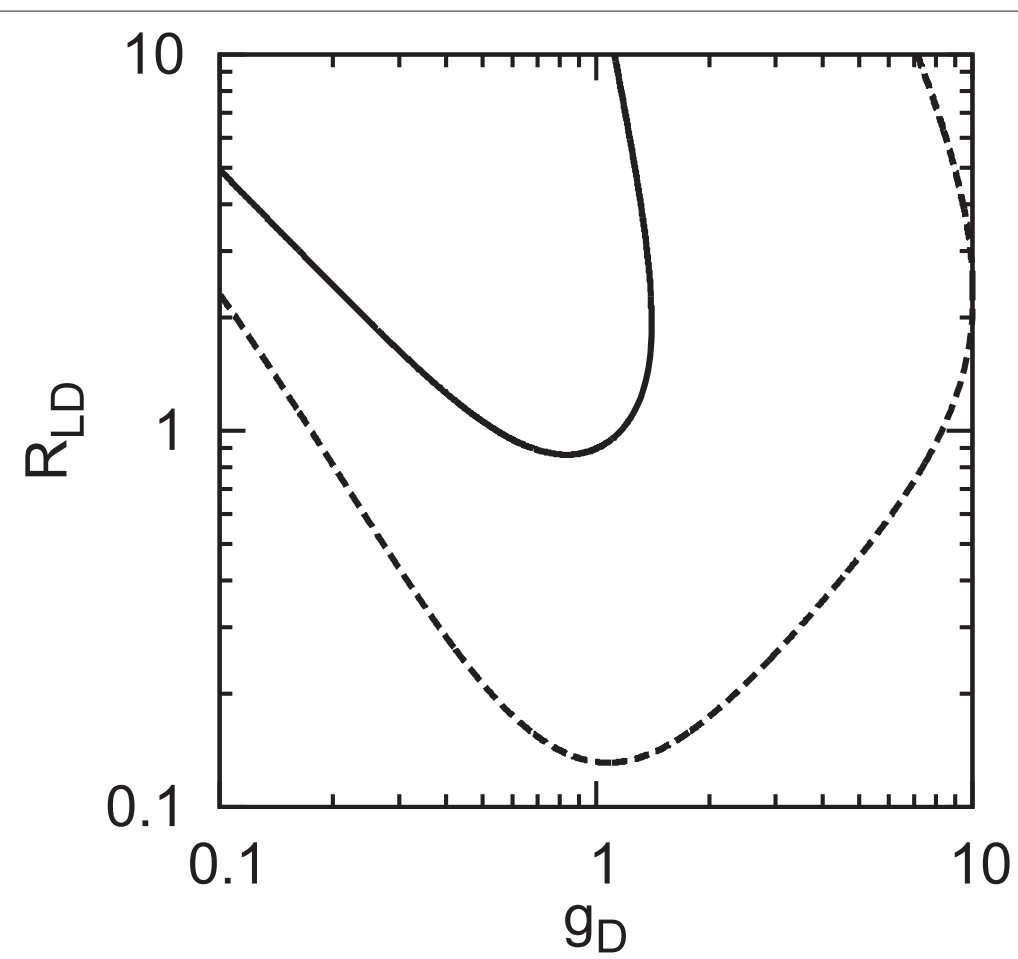

Figure 4 Contours of the averaged relative velocity of the grains are shown on the $g_{\mathrm{D}}-\boldsymbol{R}_{\mathrm{LD}}$ plane. The solid and dashed lines represent $<w_{\text {rel }}>=1$ and 0.1 , respectively.

size satisfying $\mathrm{St}=1$ at $R_{1} \sim 0.5$. Since $R_{\mathrm{LD}}$ is approximately 1 when $\alpha=10^{-4}$, we find from Figure 4 that the relative velocity $<w_{\text {rel }}>$ is approximately 1 . In this case, the relative velocity due to the lift force $\eta v_{\mathrm{K}}<w_{\text {rel }}>$ is approximately $60 \mathrm{~m} \mathrm{~s}^{-1}$, where we note that $\eta \nu_{\mathrm{K}}$ does not depend on $R_{1}$ when $p=1 / 4$. This value exceeds the relative velocity due to the turbulence $<v_{\mathrm{d}-\mathrm{d}}>{ }_{t} \sim 8 \mathrm{~m}$ $\mathrm{s}^{-1}$, which is computed with Equation (15). Therefore, the lift force is expected to efficiently affect the growth rate of the grain whose size is $10 \mathrm{~cm}$ at $R_{1} \sim 0.5$ if there is a grain-concentrated part.

Recently, a steeper density profile, which is denser at 1 $\mathrm{AU}$, has been proposed (Desch 2007). When the density profile is steeper, a larger $q$ is adopted. If $q$ is larger, the radial profile of the minimum and maximum sizes of the grains is steeper [Equation (23) and dashed green lines in Figure 2]. Moreover, we can find that the red line in Figure 2 does not change as much as the green lines by comparing Equation (16) to (20). Thus, the blue region in Figure 2 slightly shifts to the inner region. Therefore, we expect that when the density profile is steeper with the normalization coefficient fixed, the innermost radius where the lift force continues to be exerted on the grains slightly decreases close to $R_{1}=1$.

Suppose that the disk has a larger surface density. Spin-down time [Equation (20)] and Stokes number [Equation (14)] are independent of the surface density $\Sigma_{0}$.
Conversely, collision time is inversely proportional to $\Sigma_{0}$ [Equation (16)], so that $r_{\mathrm{d}}$ satisfying $t_{\mathrm{col}}=t_{\text {spin-down }}$ is proportional to $\Sigma_{0}^{-1 / 2}$ for St $\gg 1$. Additionally, $r_{\mathrm{d}, \min }$ and $r_{\mathrm{d} \text {,max }}$ in Equation (23) are inversely proportional to $\Sigma_{0}$. Therefore, the blue region moves outside and the minimum grain size in the blue region is nearly unchanged. The ratio $R_{\mathrm{LD}}$ becomes smaller with decreasing $R_{1}$, so that the relative velocity due to the lift force is smaller than that for the fiducial surface density.

\section{Model refinement for other effects}

We can refine the model in this paper by taking into account realistic porosity and shape of the dust grain (Suyama et al. 2008). If the grain is fluffy, $\rho_{\text {int }}$ is smaller than the value we used in the 'Sustainability of the spin of the dust grains' section. Equations (14), (18), and (17) lead to the dependence of $t_{\mathrm{col}}$ on $\rho_{\mathrm{int}}$,

$$
t_{\mathrm{col}} \propto \begin{cases}\rho_{\mathrm{int}}^{\frac{1}{2}} & \text { forSt } \gg 1 \\ \rho_{\mathrm{int}}^{0} & \text { forSt } \ll 1,\end{cases}
$$

while $t_{\text {spin-down }} \propto \rho_{\text {int }}^{1}$. Thus, when $\rho_{\text {int }}$ decreases, $t_{\text {spin-down }}$ decreases more rapidly than $t_{\text {col }}$, which means that the lift force is exerted on the grain over a shorter period of time. If the grain is lumpy, the coefficient of lift would become as large as a baseball or a golf ball. 
It is also worth taking into account realistic collision processes between the grains, such as simple scattering (bouncing), minor merger, and destruction. Through the simple scattering, the grain may gain the spin angular momentum by means of surface friction, where the maximum surface velocity of the scattered grains is $v_{\mathrm{d}-\mathrm{d}}$, which is less than the mean surface velocity in the case of the major merger [Equation (47)]. In addition, in the case of a minor merger, which is realized when we consider the size distribution of grains (Windmark et al. 2012), the grains obtain less spin angular momentum compared to the case of the major merger. Thus, we expect less mean relative velocity when the grains undergo the scattering and minor merger. On the other hand, if the destruction (fragmentation) occurs when the grains collide, the grains may gain larger spin angular momentum. The experiments show that agglomerates (centimeter to decimeter size) are divided into many fragments that are of millimeter to centimeter size through low-velocity collisions (Deckers and Teisere 2012; Schräpler et al. 2012). If these fragments have a large spin angular momentum, they can be sufficiently affected by the lift force. How much spin angular momentum grains gain depends on many parameters, but we will defer this question to future work.

The $z$-component of the lift force, which is omitted in this paper, may affect the resultant relative velocity of the dust grain. The equation of motion in the $z$-direction includes the gravitational force by the central star, the drag force, and the lift force. Although the steady state cannot be realized because the gravitational force depends on the altitude from the disk mid-plane, the dust grains should gain a momentum in the $z$-direction. This causes an increase in the absolute value of the velocity, which may result in an increase in the relative velocity.

\section{Conclusions}

In this paper, we investigated the effects of the lift force on dust grains in a protoplanetary disk from two perspectives. We first investigated whether the lift force is kept exerted on the grains or not. We assumed the grains are in the minimum mass solar nebula where the turbulence develops. We estimated the collision timescale and the spin-down timescale and found that the grain keeps spinning as a result of collisions with the other grains if the radius of the grain is larger than $100 \mathrm{~cm}$ at $\gtrsim 1 \mathrm{AU}$ from the central star.

Next, we calculated the mean relative velocity between the grains caused by the lift force. The grains obtain spin angular momenta with various directions by collisions between themselves, so that the lift forces exerted on them have various directions as well. Thus, the relative velocity yields between the grains. We assumed that the grains are in the steady state and that the distribution of their spin momenta shows the isotropy. We then showed that the mean relative velocity is comparable to the gas velocity at the Kepler rotational frame when $F_{\mathrm{L}} \gtrsim F_{\mathrm{D}}$ and $t_{\mathrm{s}} \approx 1 / \Omega_{\mathrm{K}}$, where $F_{\mathrm{L}}, F_{\mathrm{D}}, t_{\mathrm{s}}$, and $\Omega_{\mathrm{K}}$ are the lift force, the drag force, the stopping time of the grains by the drag, and the Kepler angular velocity, respectively. This means that the lift force can sufficiently affect the collision rate, which affects the growth rate of the grains under the parameter set.

We also estimated the mean relative velocity when the grains keep spinning by combining the above two results. We found that for the minimum mass solar nebula, the mean relative velocity due to the lift force is smaller than the gas velocity at the Kepler rotational frame. We present the mean relative velocity as being comparable to the gas velocity if the disk has grain-concentrated parts where the dust-gas ratio is ten times larger than MMSN, so the lift force may affect the collision rate in the parts.

\section{Abbreviations}

MMSN: Minimum mass solar nebula.

\section{Competing interests}

The authors declare that they have no competing interests.

\section{Authors' contributions}

MSY carried out the estimation of the mean relative velocity of the grains, participated in the sequence alignment, and drafted the manuscript. SSK carried out the estimation of the condition under which the lift force is kept exerted on the grains, participated in the sequence alignment, and drafted the manuscript. Both authors read and approved the final manuscript.

\section{Acknowledgements}

MSY and SSK thank T. Tsuribe, S. Inutsuka, and S. Okuzumi for useful discussions and comments and acknowledge the anonymous referees for useful comments as well. SSK is supported by a Grant-in-Aid from the JSPS (Japan Society for the Promotion of Science) Research Fellowships for Young Scientists program (A2517840).

\section{Author details}

${ }^{1}$ National Astronomical Observatory, 2-21-1 Osawa, Mitaka, Tokyo 181-8588, Japan. ${ }^{2}$ Department of Earth and Space Science, Graduate School of Science, Osaka University, 1-1 Machikaneyama, Toyonaka, Osaka 560-0043, Japan.

Received: 30 April 2014 Accepted: 23 September 2014

Published: 3 October 2014

\section{References}

Adachi I, Hayashi C, Nakazawa K (1976) The gas drag effect on the elliptical motion of a solid body in the primordial solar nebula. Prog Theor Phys 56:1756-1771

Balbus SA, Hawley JF (1976) A powerful local shear instability in weakly magnetized disks. I - Linear analysis. II - Nonlinear evolution. Astrophys J 376:214-233

Birnstiel T, Dullemond CP, Brauer F (2010) Gas- and dust evolution in protoplanetary disks. Astron Astrophys 513:79-99

Brauer F, Dullemond CP, Henning Th (2008) Planetesimal formation near the snow line in MRI-driven turbulent protoplanetary disks. Astron Astrophys 487:1-4

Deckers J, Teiser J (2012) Colliding decimeter dust. Astrophys J 769:151-156

Desch SJ (2007) Mass distribution and planet formation in the solar nebula. Astrophys J 671:878-893

Goldreich P, Ward W (1973) The formation of planetesimals. Astrophys J 183:1051-1062

Hasegawa Y, Tsuribe T (2014) Kelvin-Helmholtz instabilities in multi-sized dust layers. Publ Astron Soc Jpn 66:L2 
Hayashi C (1981) Structure of the solar nebula, growth and decay of magnetic fields and effects of magnetic and turbulent viscosities on the nebula. Prog Theor Phys Suppl 70:35-53

Kataoka A, Tanaka H, Okuzumi S, Wada K (2013) Fluffy dust forms icy planetesimals by static compression. Astron Astrophys 557:L4

Okuzumi S, Tanaka H, Kobayashi H, Wada K (2012) Rapid coagulation of porous dust aggregates outside the snow line: a pathway to successful icy planetesimal formation. Astrophys J 752:106-123

Ormel CW, Cuzzi JN (2007) Closed-form expressions for particle relative velocities induced by turbulence. Astron Astrophys 466:413-420

Rubinow SI, Keller JB (1961) The transverse force on a spinning sphere moving in a viscous fluid. J Fluid Mech 11:447-459

Sano T, Miyama SM, Umebayashi T, Nakano T (2000) Magnetorotational instability in protoplanetary disks. II. Ionization state and unstable regions, Astrophys J 543:486-501

Schräpler R, Blum J, Seizinger A, Kley W (2012) The physics of protoplanetesimal dust agglomerates. VII. The low-velocity collision behavior of large dust agglomerates. Astrophys J 758:35-43

Sekiya M (1983) Gravitational instabilities in a dust-gas layer and formation of planetesimals in the solar nebula. Prog Theor Phys 69:1116-1130

Sekiya, M (1998) Quasi-equilibrium density distributions of small dust aggregations in the solar nebula. Icarus 133:298-309

Shirayama S (1992) Flow past a sphere: topological transitions of the vorticity field. AIAA J 30:349-358

Suyama T, Wada K, Tanaka H (2008) Numerical simulation of density evolution of dust aggregates in protoplanetary disks. I. Head-on collisions. Astrophys J 684:1310-1322

Takagi H (1974) On the slow motion of a sphere in a viscous fluid. J Phys Soc Jpn 37:505-510

Windmark F, Birnstiel T, Güttler C, Blum J, Dullemond CP, Henning Th (2012) Planetesimal formation by sweep-up: how the bouncing barrier can be beneficial to growth. Astron Astrophys 540:73-89

Youdin AN, Goodman J (2005) Streaming instabilities in protoplanetary disks. Astrophys J 620:459-469

doi:10.1186/1880-5981-66-132

Cite this article as: Yamaguchi and Kimura: Effect of lift force on the aerodynamics of dust grains in the protoplanetary disk. Earth, Planets and Space 2014 66:132.

\section{Submit your manuscript to a SpringerOpen ${ }^{\mathcal{O}}$ journal and benefit from:}

- Convenient online submission

Rigorous peer review

- Immediate publication on acceptance

- Open access: articles freely available online

- High visibility within the field

- Retaining the copyright to your article

Submit your next manuscript at $>$ springeropen.com 